\title{
Substrate Templating upon Self-Assembly of Hydrogen- Bonded Molecular Networks on an Insulating Surface
}

\author{
Philipp Rahe, * Markus Nimmrich, and Angelika Kühnle
}

\begin{abstract}
Molecular self-assembly on insulating surfaces, despite being highly relvant to many applications, generally suffers from the weak molecule-surface interactions present on dielectric surfaces, especially when benchmarked against metallic substrates. Therefore, to fully exploit the potential of molecular self-assembly, increasing the influence of the substrate constitutes an essential prerequisite. Upon deposition of terephthalic acid and trimesic acid onto the natural cleavage plane of calcite, extended hydrogen-bonded networks are formed, which wet the substrate. The observed structural complexity matches the variety realized on metal surfaces. A detailed analysis of the molecular structures observed on calcite reveals a significant influence of the underlying substrate, clearly indicating a substantial templating effect of the surface on the resulting molecular networks. This work demonstrates that choosing suitable molecule/substrate systems allows for tuning the balance between intermolecular and molecule-surface interactions even in the case of typically weakly interacting insulating surfaces. This study, thus, provides a strategy for deliberately exploiting substrate templating to increase the structural variety in molecular selfassembly on a bulk insulator at room temperature.
\end{abstract}

\section{Introduction}

Molecular self-assembly constitutes a versatile strategy for creating functional structures at surfaces. ${ }^{[1]}$ In the last decades, a wide range of molecular architectures have been obtained by tuning the subtle balance between intermolecular and molecule-surface interactions on metallic surfaces, ${ }^{[2]}$ including complex hydrogen-bonded network structures. ${ }^{[3-5]}$ Especially when having molecular electronics applications in mind, however, electrically insulating dielectrics rather than conducting metallic surfaces are mandatory for electronically

Dr. P. Rahe, ${ }^{[+]}$M. Nimmrich, Prof. A. Kühnle Institut für Physikalische Chemie Johannes Gutenberg-Universität Mainz Duesbergweg 10-14, 55099 Mainz, Germany E-mail: rahe@uni-mainz.de

[+] Current address: Department of Physics \& Astronomy, University of Utah, 115 South 1400 East, Salt Lake City, UT 84112, USA

DOI: $10.1002 /$ smll.201200681 decoupling molecular structures, such as molecular wires, from the underlying support.

So far, the weak binding energy and the small diffusion barrier of organic molecules on prototypical bulk insulator surfaces, such as $\mathrm{KBr}(001), \mathrm{NaCl}(001), \mathrm{KCl}(001)$ or insulating thin films, have largely hampered the exploitation of molecular self-assembly principles for insulating support systems at room temperature. Due to the weak molecular-surface interaction and the resulting high molecular mobility, clustering at step edges ${ }^{[6,7]}$ and molecular bulk formation ${ }^{[8]}$ are frequently observed, both prohibiting surface-templated molecular structures to be obtained. As an illustration for the weak molecule-surface interactions, stable hydrogen-bonded networks formed by melamine and cyanuric acid have only been realized to be stable on an $\mathrm{NaCl}$ thin film when reducing the mobility upon cooling to $160 \mathrm{~K} .^{[9]}$

Additionally, commensurate phases of perylene-3,4,9,10tetracarboxylic dianhydride (PTCDA) on $\mathrm{KCl}(001)$ and $\mathrm{NaCl}(001)$ surfaces have been presented at room temperature. ${ }^{[10,11]}$ However, these structures were observed to be metastable as demonstrated by increasing the coverage or substrate temperature, which readily resulted in the formation 
of the bulk phase. Only very recently, an elegant example for an extended hydrogen-bonded network has been presented, utilizing the unusually strong intermolecular cohesion energy of boronic acid to form a wetting layer on $\mathrm{KBr}(001) .{ }^{[12]}$ In the latter study, however, the substrate has been demonstrated to have marginal influence on the resulting molecular film, as further corroborated by the low desorption temperature of around $370 \mathrm{~K}$.

To grasp the full potential of molecular self-assembly, a significant templating effect of the substrate is necessary to increase structural variety. In terms of molecule-surface interactions, the natural cleavage plane of calcite, $\mathrm{CaCO}_{3}(10 \overline{1} 4)$, has been demonstrated to constitute a promising insulating substrate for molecular self-assembly due to its high surface energy. ${ }^{[13]}$

Moreover, the comparable high binding energy of about $1 \mathrm{eV}$ of carboxylic acid moieties towards the calcite surface ${ }^{[14]}$ has been utilized to confine halogenated benzoic acid molecules to the surface, even during thermal annealing for an onsurface covalent linking of the molecules. ${ }^{[15]}$ Here, we chose planar organic molecules with carboxylic acid groups providing the possibility for both increased interaction with the underlying substrate and intermolecular hydrogen bond formation. Two aromatic carboxylic acids are studied within this work, namely terephthalic acid (TPA, $\mathrm{C}_{8} \mathrm{O}_{4} \mathrm{H}_{6}$ ) and trimesic acid (TMA, $\mathrm{C}_{9} \mathrm{O}_{6} \mathrm{H}_{6}$ ). A schematic representation of both molecules is given in Figure 1. TPA consists of a central benzene with two carboxylic acid groups in a para configuration, while TMA has a central benzene ring with three carboxylic

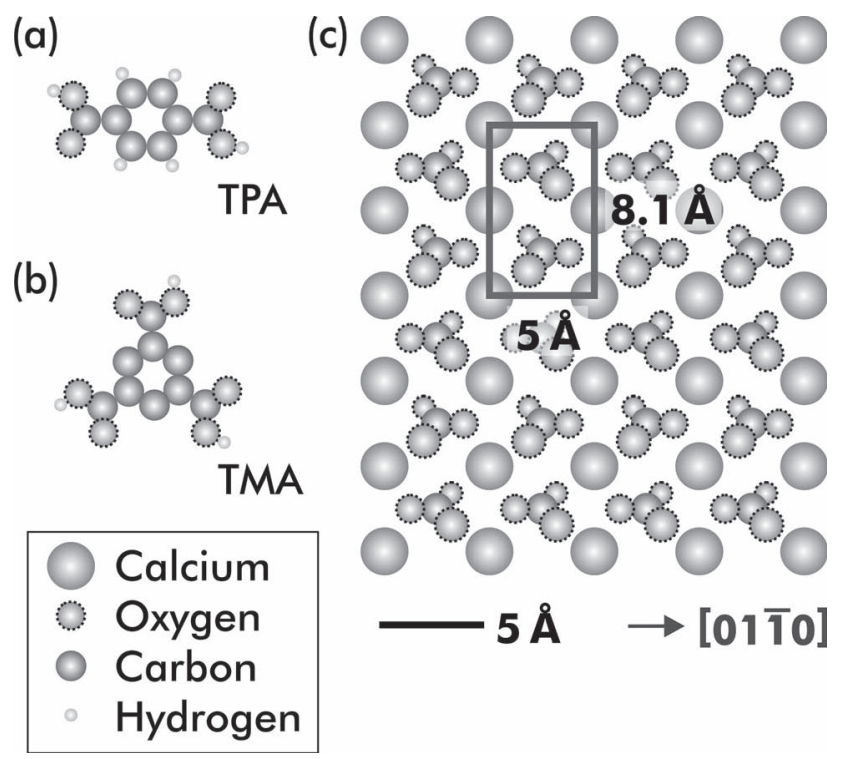

Figure 1. Model of the a) terephthalic acid (TPA) molecule, b) trimesic acid (TMA) molecule, and c) the $\mathrm{CaCO}_{3}(10 \overline{1} 4)$ surface. The scale bare applies to all subfigures. The $\mathrm{CaCO}_{3}(10 \overline{1} 4)$ surface has a rectangular unit cell of size $5.0 \AA \times 8.1 \AA$ consisting of two carbonate groups and two calcium ions. The carbonate groups are rotated such that one oxygen lies above, one in, and one below the plane spanned by the calcium ions. Furthermore, the different orientation of the two carbonate groups leads to a characteristic zigzag pattern of the topmost oxygen atoms. The existence of surface reconstructions has been discussed recently. ${ }^{[16-19]}$ acid groups attached. In the case of the TPA/ $\mathrm{CaCO}_{3}(10 \overline{1} 4)$ system (see Section 2.1), extended structures of hydrogenbonded ribbons are revealed at room temperature, which resemble part of the molecular bulk motif. Compared to the crystallographic bulk structure, however, the hydrogen bonds in the $\mathrm{TPA} / \mathrm{CaCO}_{3}(10 \overline{1} 4)$ structure are somewhat stretched, and the resulting molecular chains are considerably separated, indicating the templating influence of the underlying substrate.

For TMA/CaCO $3(10 \overline{1} 4$ ) (see Section 2.2), a variety of coexisting structures is obtained. Interestingly, the basic motif of the TMA bulk structure, the so-called chicken-wire structure, is only revealed in fragments covering tiny surface areas. The absence of an extended chicken-wire phase directly demonstrates the incommensurability of the bulk structure with the calcite lattice. In contrast, the so-called flower structure, where the TMA molecules arrange in a close-packed fashion, is observed in extended areas. Interestingly, this motif is compressed on calcite compared to the common high-symmetry configuration, thus again indicating an intense templating effect of the calcite substrate.

By exploiting substrate templating, this study paves the way for molecular self-assembly on an insulating surface even at room temperature.

\section{Results and Discussion}

\subsection{Terephthalic Acid (TPA)}

Terephthalic acid (TPA) is a versatile molecular linker and has, consequently, received considerable attention with respect to the assembly of $3 \mathrm{D}$ self-assembled porous frameworks stabilized by metal-carboxylate bonds ${ }^{[20]}$ as well as in 2D supramolecular architectures at surfaces. ${ }^{\text {[21-28] }}$

Bailey and Brown ${ }^{[29]}$ have investigated the TPA bulk structure by means of X-ray diffraction. From their data, two triclinic forms have been identified, both presumably of space group $P \overline{1}$. For both forms, chains of TPA molecules linked via hydrogen bonds between two carboxylic acid groups are reported as the basal unit. Along these molecular chains, the molecule-molecule distance has been measured to $9.54 \AA$ while the chain separation is in the range of 5.2 to $5.3 \AA$ depending on the polymorph. ${ }^{[29]}$

Obviously, hydrogen-bonded linking via the carboxylic acid groups is the common motif found when studying the TPA superstructures on substrates such as highly ordered pyrolytic graphite (HOPG), ${ }^{[21]} \mathrm{Pd}(111),{ }^{[25]}$ or $\mathrm{Au}(111) .{ }^{[26]}$ The structure formation changes substantially when the TPA molecules are deprotonated, which has, for example, been presented in experiments on $\operatorname{Pd}(111)^{[25]}$ and $\mathrm{Cu}(100) .{ }^{[22]}$ Upon strong interaction with the substrate, however, the molecular assembly of TPA has often been observed to be dictated by the surface structure. To name a prominent example, a full monolayer of TPA molecules on a $\mathrm{TiO}_{2}(110)$ substrate favors an upright orientation as has first been observed by Tekiel et al. ${ }^{[30]}$ Later, it was shown in a combined non-contact atomic force microscopy (NC-AFM) and near-edge X-ray 

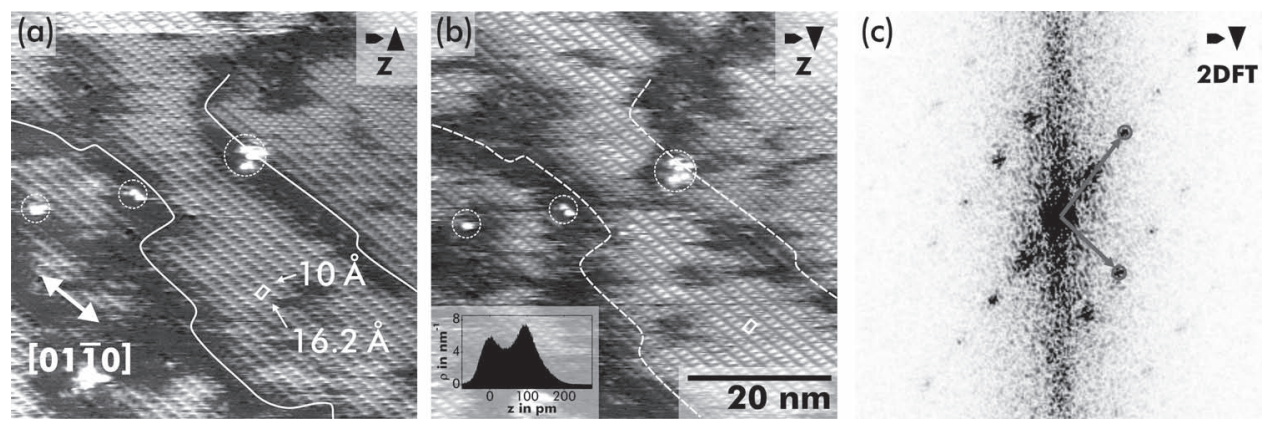

Figure 2. a,b) Consecutive AFM images taken at a sample temperature of $300 \mathrm{~K}$. Three immobile features are marked as a reference. The edges of two island structures in (a) are highlighted. c) Central part of the 2D Fourier transform (FT) taken from image (b).

absorption fine structure (NEXAFS) experimental study that a structural transition exists from flat-lying molecules at sub-monolayer coverages to upright-standing molecules at monolayer coverage. ${ }^{[27]}$

First, a submonolayer coverage ${ }^{[31]}$ of TPA is deposited on a clean $\mathrm{CaCO}_{3}(10 \overline{14})$ surface at room temperature. After TPA deposition, the sample is transferred to the AFM. Two consecutive images are presented in Figure 2a and b (acquisition time per image $\sim 8.5 \mathrm{~min}$ ).

At this coverage of less than a monolayer, islands with fuzzy edges are observed. Additionally, a moderate molecular mobility is apparent as the island edges reorganize. To visualize this island reorganization, two of the island edges
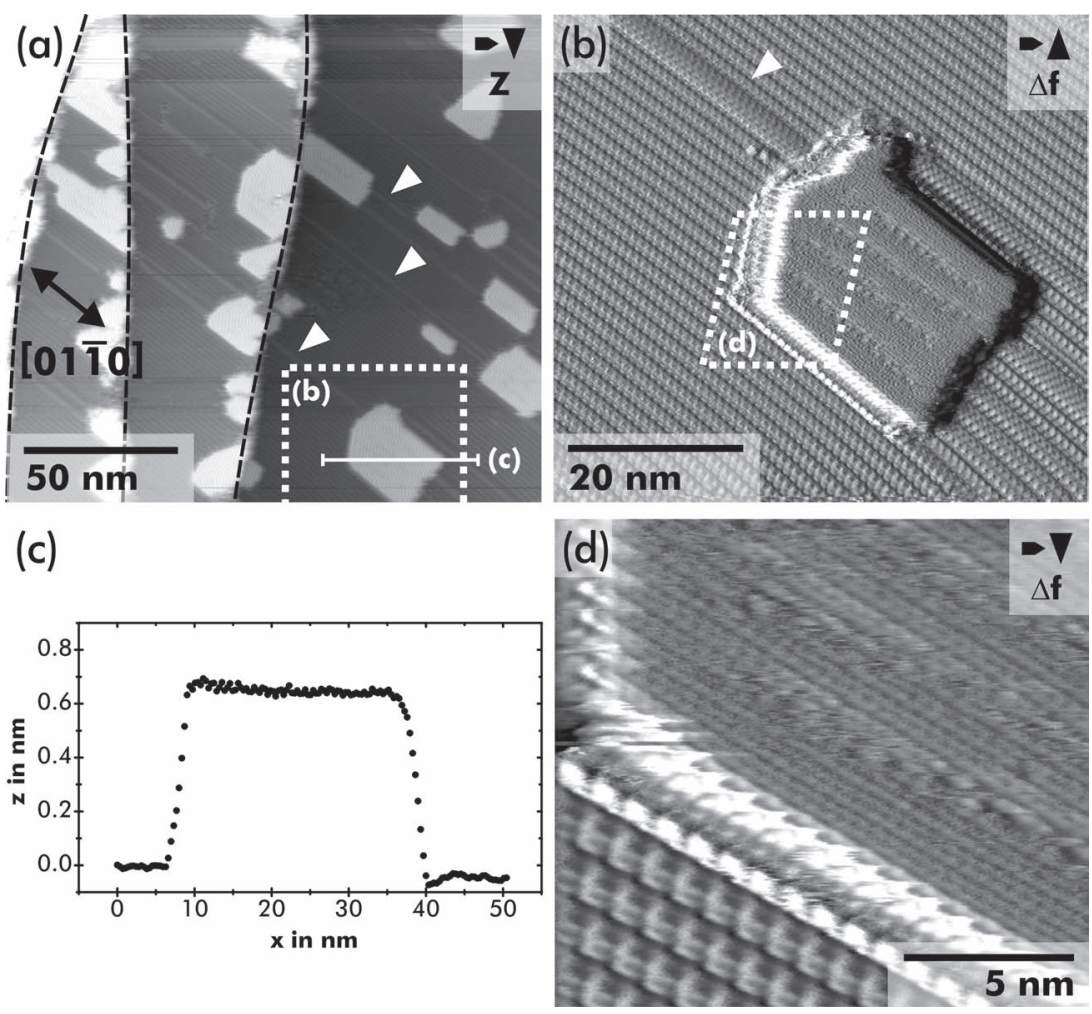

Figure 3. a) Immobile TPA superstructure imaged on a $\mathrm{CaCO}_{3}(10 \overline{1} 4)$ surface. The structure is stabilized by depositing slightly more than one monolayer of TPA molecules. b,d) Detailed images acquired at the positions indicated in (a) and (b). c) Line profile across the second phase extracted as indicated in (a). are highlighted in Figure 2a by white lines. These edges are reproduced ${ }^{[32]}$ in Figure $2 b$ and, thus, directly map the edge reconfiguration. This observation indicates i) a high molecular mobility at room temperature and ii) the fact that individual molecules can detach from an island at room temperature.

The inner structure of these islands bears a well-defined pattern. From several 2D Fourier transform (2D-FT) data (one shown in Figure 2c), a rectangular unit cell of size $(10.0 \pm 0.2) \AA \times(16.3 \pm 0.4) \AA$ is measured. This unit cell, which is also indicated in the real-space domain in Figure 2a and $b$, is in excellent agreement with a $(2 \times 2)$ superstructure, having a unit cell size of $10.0 \AA \times 16.2 \AA$.

The diffusion of TPA molecules limits the high-resolution capabilities of the NC-AFM technique. This is circumvented by either immobilizing the molecules at reduced temperatures or upon forming a full monolayer. Figure 3 presents NC-AFM data where the latter strategy is followed. A molecular coverage of slightly more than one monolayer is deposited, and, as is directly apparent from the image, the molecular structure becomes immediately immobile.

The overview NC-AFM image in Figure 3 clearly suggests a layer-by-layer growth mode for TPA molecules on the $\mathrm{CaCO}_{3}(10 \overline{1} 4)$ surface, the calcite substrate is completely covered.

Predominantly, the TPA molecules arrange in a $(2 \times 2)$ superstructure, which will be analyzed in detail in the following.

Interestingly, a second phase coexists, forming molecular islands of increased height. This second phase mainly nucleates at calcite step edges (step edges highlighted by black dashed lines in Figure 3a); however, several islands are also observed aside step edges as apparent on the lowest terrace in Figure 3a. Our NC-AFM data reveal that these islands appear to nucleate at the ending of dense stripes in the $(2 \times 2)$ structure. Some of these dense stripes, which will later be identified as 
domain boundaries in the $(2 \times 2)$ structure, are marked by white arrows in Figure $3 a$ and $b$.

The island height of the second phase is measured in Figure 3 to $7 \pm 1 \AA$ relative to the TPA monolayer (see line profile in Figure 3c). Although the absolute height measured with the NC-AFM technique is questionable, especially in the presence of charge transfer to or from adsorbed molecules, ${ }^{[33]}$ the apparent height measured here is in between the expected dimensions of flat-lying $(\sim 3 \AA)$ and uprightstanding ( $10 \AA)$ TPA molecules. Therefore, it cannot be clarified unambiguously whether the second phase is formed by a second molecular layer or by upright-standing molecules embedded in the first $(2 \times 2)$ layer. However, the focus in this work will exclusively lie on the predominant $(2 \times 2)$ phase.

Figure 4a presents a high-resolution image from a part of the data shown in Figure $3 \mathrm{a}$. The $(2 \times 2)$ superstructure already observed in Figure 2, where a submonolayer coverage of TPA was deposited, is confirmed from these data acquired at a full monolayer coverage. Consequently, no coverage dependence of the adsorption structure is revealed for this $(2 \times 2)$ phase and discussing the full monolayer structure is expected to entirely cover the structure formed in the submonolayer regime.

The detailed view at the molecular structure in Figure 4a reveals molecular double chains oriented along the [1010] substrate direction. Each of these chains is supposed to be formed by flat-lying TPA molecules. Additionally, our data suggest that the molecules along one chain are linked by hydrogen bonds between two carboxylic acid groups as visualized in the structural model in Figure $4 \mathrm{~b}$. The observed periodicity of $10 \AA$ fits excellently to this geometry, it is agreement to the two-fold superstructure along $a$, and furthermore, it is close to the distance observed in the TPA bulk structure. Compared to the bulk structure, ${ }^{[29]}$ however, the hydrogen bonds in the TPA/CaCO $3(10 \overline{1} 4)(2 \times 2)$ superstructure are slightly stretched by $\sim 45 \mathrm{pm}$ due to a lattice mismatch of $\sim 5 \%$ between the TPA bulk and the $\mathrm{CaCO}_{3}(10 \overline{1} 4)(2 \times 2)$ superstructure dimensions. The formation of the hydrogen bonds requires protonated molecules, which is in agreement with the molecule's $\mathrm{p} k_{\mathrm{a}}$ value. ${ }^{[15,34]}$

Usually, two adjacent chains are observed to pair to a molecular ribbon. One molecular ribbon is depicted in Figure 4b, where two TPA chains are linked perpendicular to the chain direction. These ribbons align with a periodicity of $16.2 \AA$ along the [ $\overline{42} 61]$ direction, according to the two-fold superstructure along $b .^{[35]}$

The interchain interaction can be explained by a combination of weak hydrogen bonding between the carboxylic acid dimers and the hydrogen atoms at the benzene core as well as weak van-der-Waals forces between the involved molecules. Compared to the situation in the bulk, ${ }^{[29]}$ where two TPA chains have a periodicity in the range of 10.4 to $10.6 \AA$ depending on the inter-chain shift, a significant influence of the calcite substrate is present, as the chains do not assemble in the most dense, but in a rather open fashion.

The data in Figure 5a, which are another zoom into Figure 3a, reveal two more characteristics of the $(2 \times 2)$ phase, which were not identified at submonolayer coverages. First, two domains for the $(2 \times 2)$ superstructure are revealed, referred
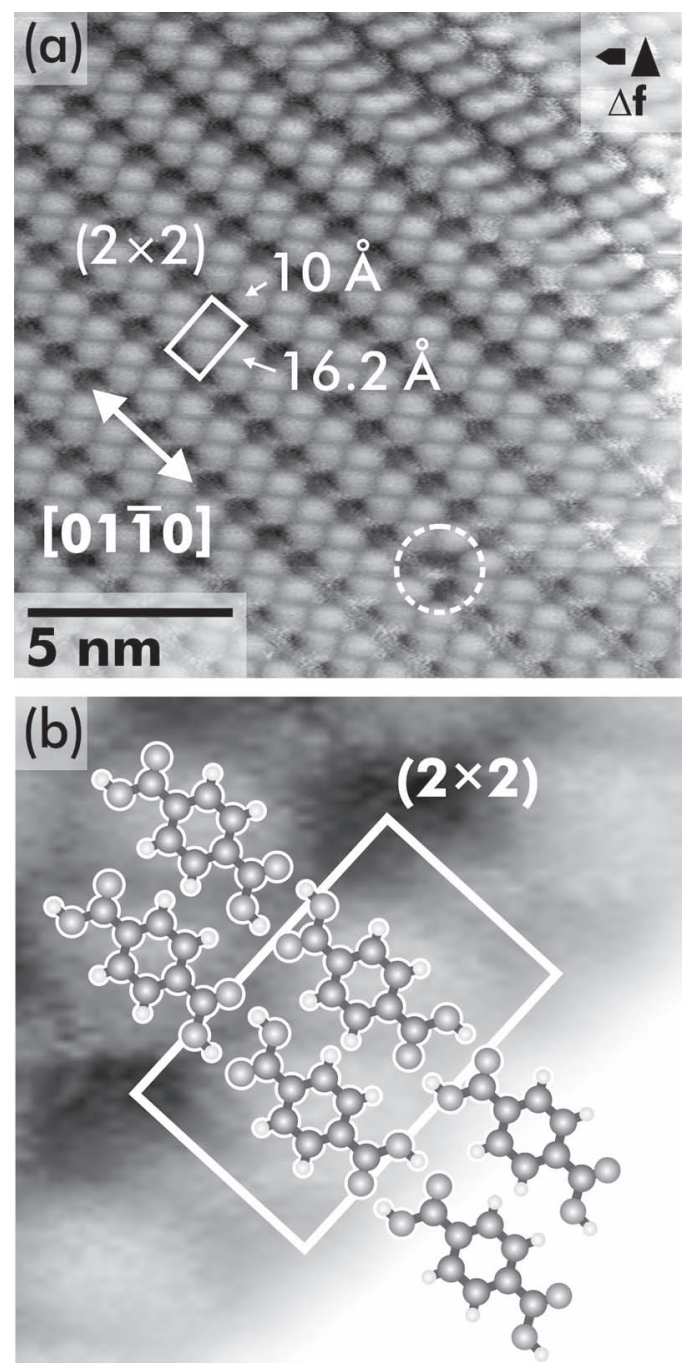

Figure 4 . Structure of the TPA $(2 \times 2)$ superstructure on a $\mathrm{CaCO}_{3}(10 \overline{1} 4)$ surface imaged at room temperature. a) Detailed image including a single-molecular defect (marked by a dashed white circle). b) Structural model of the molecular ribbon, consisting of two chains of TPA molecules. Each chain forms the basic motif in the TPA bulk structure.

to as $\alpha$ and $\beta$. The two domains differ in their appearance due to a different chain orientation inside the molecular ribbons as will be analyzed later. Second, these domains are separated by boundaries formed by densely packed TPA molecules. Two alternatives of these boundaries are possible and observed experimentally: one type separates two equal domains (herein named type $1_{\alpha-\alpha}$ and type $1_{\beta-\beta}$ ), while the second separates two different domains (type $2_{\alpha-\beta}$ ).

The periodicity of the molecular structure along the [01 $\overline{1} 0]$ direction inside the domain boundaries agrees well with the $(2 \times 2)$ superstructure along this direction, and thus, the domain boundaries are most likely formed by dense arrangements of TPA chains.

For analyzing the structure of domains $\alpha$ and $\beta$, it is instructive first to measure the width of the domain boundaries. This width is equivalent to the separation of the TPA ribbons across the respective boundary. For the two boundaries 

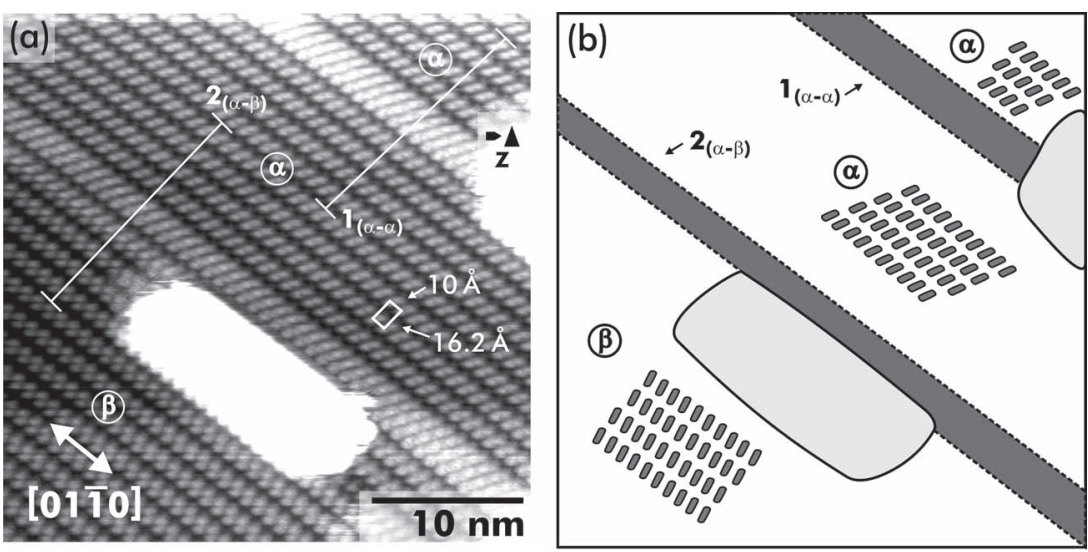

Figure 5. a) Domain boundary structure revealed for the $\mathrm{TPA} / \mathrm{CaCO}_{3}(10 \overline{1} 4)$ structure. b) Schematic representation of the NC-AFM image in (a), revealing two domains of the $(2 \times 2)$ superstructure ( $\alpha$ and $\beta$ ) as well as two domain boundaries 1 and $\mathbf{2}$.

marked in Figure 5a, averaged line profiles extracted perpen-

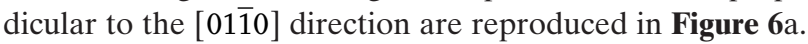

According to the $(2 \times 2)$ superstructure, the periodicity perpendicular to the [0110] direction is $16.2 \AA$. This periodicity

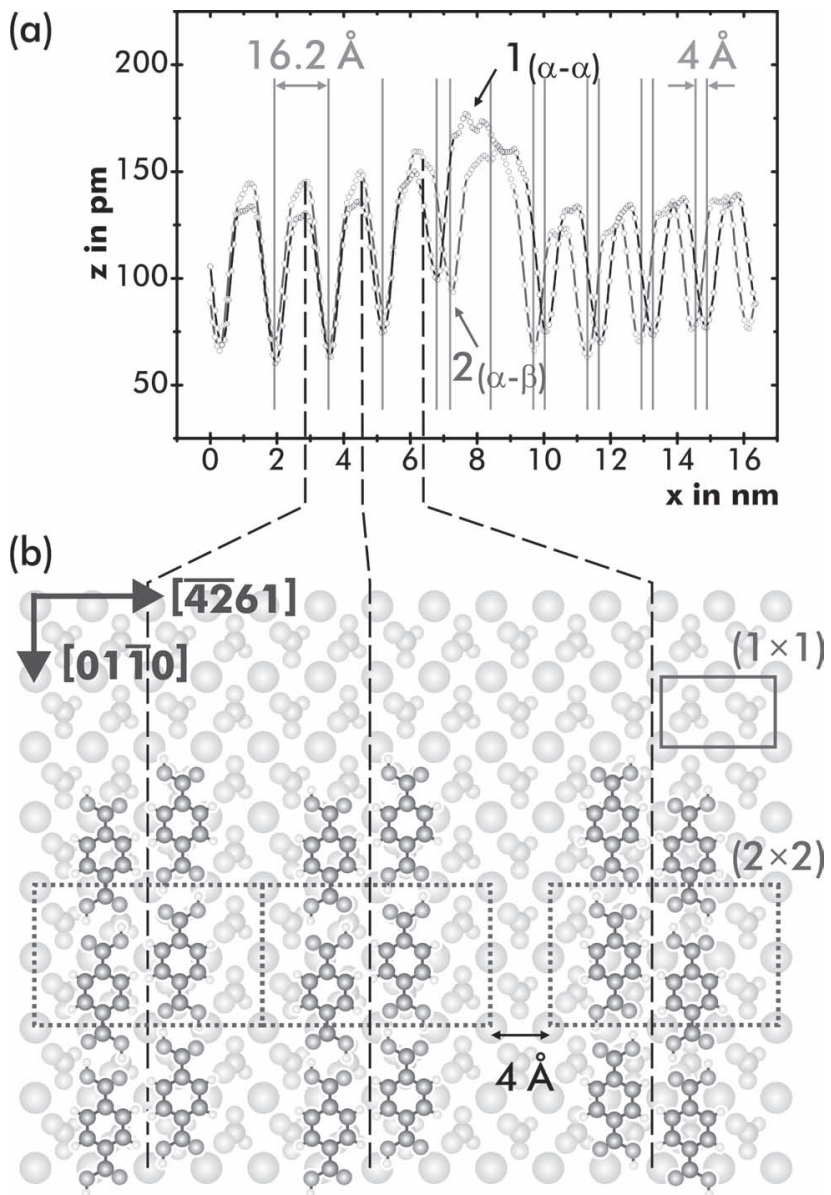

Figure 6. a) Two averaged line profiles from Figure $5 a$. Profile 1 is extracted across a boundary with adjacent $\alpha-\alpha$ domains, while profile 2 separates domains $\alpha-\beta$. b) Refined adsorption model for the $(2 \times 2)$ superstructure. For clarity reasons, the embedded single chain visible in Figure 5 a is not reproduced in the $4 \AA$ gap. is visualized by vertical gray lines in Figure 6a. Interestingly, the lines match the molecular arrangement across boundary $1_{\alpha-\alpha}$, while for boundary $2_{\alpha-\beta}$, a shift of about $4 \AA$ is found in these data.

This shift at the $\alpha-\beta$ boundary is in excellent agreement with a shift by $b / 2$, where $b$ is the $\mathrm{CaCO}_{3}(10 \overline{1} 4)$ unit cell size perpendicular to the $[01 \overline{1} 0]$ direction. It is, thus, identical to the distance of two neighboring topmost oxygen atoms perpendicular to the $[01 \overline{1} 0]$ direction.

From this analysis, the structural model for the $(2 \times 2)$ superstructure is refined as proposed in Figure $6 \mathrm{~b}$. Two of the TPA chains are observed to pair to a molecular ribbon by weak inter-chain interaction. In this configuration, the chains are slightly shifted against each other. The shift of the "right" chain with respect to the "left" one (see Figure 6b) can either be positive or negative, leading to the structure of domains $\alpha$ and $\beta$. The fact that we observe the different domains including different domain boundaries impressively represents the templating influence of the calcite substrate.

\subsection{Trimesic Acid (TMA)}

In the following, we aim at increasing both, the intermolecular as well as the molecular-surface interaction, by introducing a third carboxylic group. The molecule chosen is trimesic acid (TMA, $\mathrm{C}_{9} \mathrm{O}_{6} \mathrm{H}_{6}$ ), a schematic representation is given in Figure $1 \mathrm{~b}$.

The bulk structure of TMA has been investigated by Duchamp and Marsh. ${ }^{[36]}$ From X-ray diffraction data they found monoclinic crystals of space group $C 2 / c$ with $a=26.52$ $\AA, b=16.42 \AA, c=26.55 \AA$, and $\beta=91.53^{\circ}$. The basic structural motif has been found as a continuous, $2 \mathrm{D}$ network consisting of large rings formed by six TMA molecules. The stabilization of this chicken-wire motif is provided by pairwise hydrogen bonds between the carboxylic acid groups.

TMA has been discussed as a molecular building block for highly ordered structures on conducting surfaces such as $\mathrm{HOPG}(0001),{ }^{[37-39]} \mathrm{Cu}(100),{ }^{[40]} \mathrm{Au}(111),{ }^{[41]}$ and $\mathrm{Ag} /$ $\operatorname{Si}(111)-(\sqrt{3} \times \sqrt{3}) \mathrm{R} 30^{\circ} .{ }^{[42]}$ On these surfaces, flat-lying TMA molecules form two characteristic structures known as the flower and chicken-wire motif. Both structures are stabilized by hydrogen bonds between the carboxylic acid groups; however, the number of carboxylic acid groups involved in the bond formation differs. While the chicken-wire structure is stabilized by carboxylic acid dimers and is, thus, equivalent to the basic bulk motif, the flower structure involves an interaction between three carboxylic acid groups. The latter structure allows a denser packing of TMA molecules compared to the chicken-wire motif.

Ye et al. ${ }^{[41]}$ investigated the self-assembly of TMA on $\mathrm{Au}(111)$ surfaces, where the evolution of the chickenwire motif to the densely packed flower structure has been reported to depend on the molecular coverage. Consequently, 

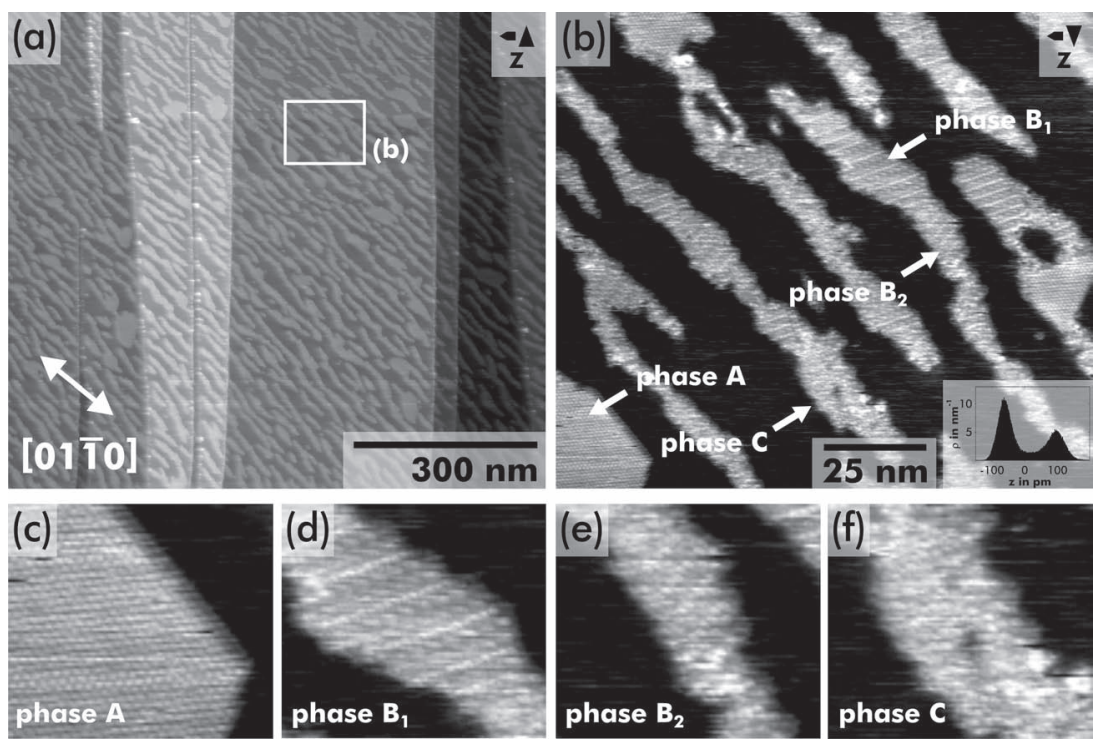

Figure 7. NC-AFM data for the $\mathrm{TMA} / \mathrm{CaCO}_{3}(10 \overline{1} 4)$ system. a) Molecular strands roughly oriented along the $[01 \overline{1} 0]$ substrate direction are observed. b) A total of four phases coexist and are labeled in this zoom-in. c) Detailed view of phase A. d) Detailed view of phase $B_{1}$. e) Detailed view of phase $B_{2}$. f) Detailed view of phase $C$.

we tested the probability of the TMA structure formation on $\mathrm{CaCO}_{3}(10 \overline{1} 4)$ to show a coverage dependency. Our experiments did not yield any indication for a coverage-dependent change in the resulting molecular structure (see Supporting Information).

Figure 7 shows representative NC-AFM data after the deposition of $\sim 0.4$ monolayer (ML) of TMA on a clean $\mathrm{CaCO}_{3}(10 \overline{1} 4)$ surface. In Figure 7a, molecular strands roughly oriented along the [01̄10] substrate direction are observed. Several nearly vertically oriented step edges of the underlying calcite substrate are seen in the image. Figure $7 b$ presents a detailed view at the position indicated in Figure $7 \mathrm{a}$. In this figure, a total of four phases coexist inside the molecular strands. One phase (phase A, Figure 7c) reveals a nearly hexagonal pattern, while phases $B_{1}$ (Figure $7 d$ ) and $B_{2}$ (Figure 7e) represent a stripe-like ordering. The fourth phase, namely phase $C$ (Figure 7f), appears first-hand unordered, but later data indicate that this structure, similar to $\mathrm{B}_{1}$ and $\mathrm{B}_{2}$, is formed by fragments of the bulk-like chicken-wire motif.

The imaged height for all molecular phases is in the order of $2 \AA$ as apparent from the height distribution (see inset Figure 7b). This finding suggests a flat-lying geometry of TMA molecules for all observed phases.

Phase A: We first discuss the nearly hexagonal ordered phase A. Figure 8a presents a high-resolution frequency shift $(\Delta f)$ image of one island where both, the molecular structure and the underlying $\mathrm{CaCO}_{3}(10 \overline{1} 4)$ surface is resolved. In contrast to the results found for TPA, the island structure shown here for TMA does not exhibit fuzzy edges, demonstrating the success of increasing the intermolecular and moleculesurface interaction by introducing a third carboxylic group. To measure lateral dimensions of the molecular structure, the presented data are carefully corrected for thermal drift. ${ }^{[43]}$ 2D-FT data from several consecutive images are taken and an exemplary 2D-FT is included in Figure 8a, where the spots originating from the molecular structure are marked by solid circles, and spots from the substrate lattice periodicity are marked by dotted circles. The former spots yield a periodicity of $d_{1}=(8.5 \pm 0.3) \AA, d_{2}$ $=(8.3 \pm 0.3) \AA$, and $d_{3}=(8.1 \pm 0.3) \AA$ for the molecular structure. For a hexagonal lattice, the nearest-neighbor distance $c_{\mathrm{i}}$ is given by $c_{\mathrm{i}}=2 / \sqrt{ } 3 \times d_{\mathrm{i}}$ from the lattice plane distances $d_{\mathrm{i}}$. Consequently, for the preceding data an average nearest-neighbor distance of $\bar{c}=(9.6 \pm 0.6) \AA$ is calculated. From the substrate spots, we reveal a unit cell size of $(4.8 \pm 0.2) \AA \times(8.4 \pm 0.4) \AA$, in good agreement with the expected crystallographic bulk data.

The average nearest-neighbor distance of the molecular structure is in excellent agreement with a nearly hexagonal superstructure on the $\mathrm{CaCO}_{3}(10 \overline{1} 4)$ surface shown in Figure 8b, again manifesting the substrate templating effect. Although atomic resolution is achieved in the image presented in Figure 8a, the absolute adsorption position is difficult to clarify as identifying the molecular core is ambiguous in NC-AFM data. However, positioning the carboxylic acid groups atop protruding surface oxygen atoms appears reasonable considering electrostatic aspects. The slightly distorted hexagonal cell of the flower motif is a commensurate superstructure, resulting in a slightly compressed hexagon. To be precise, the structure constitutes an oblique unit cell with $a_{1}=9.98 \AA, a_{2}=9.51 \AA$, and $\alpha=$ $58.35^{\circ}$. Using Wood's notation, the molecules form a $c(2 \times 2)$ superstructure. In this superstructure, the difference in the nearest-neighbor distances amounts to about $5 \%$. The dimensions are in excellent agreement with the measured distances $d_{\mathrm{i}}$ from the 2D-FT.

In Figure 8c, structural models of TMA molecules are superimposed on the oblique unit cell. The structure is expected to be stabilized by hydrogen bonds between the carboxylic acid groups of three TMA molecules and, thus, corresponds to the close-packed flower motif. This bond configuration requires protonated TMA molecules. The repeating unit is, indeed, observed in the $\Delta f$ images and even defects (one marked in the lower right of Figure 8a) are observed.

Phases $B_{1}, B_{2}$, and $C$ : The other three observed phases, namely the striped phases $B_{1}$ and $B_{2}$ as well as phase $C$, are most likely fragments of the chicken-wire motif. The chickenwire motif, which forms the basic bulk motif, is stabilized by hydrogen bonds between carboxylic acid dimers as depicted in Figure 9. Most interestingly, an extended chicken-wire motif itself is not observed on a $\mathrm{CaCO}_{3}(10 \overline{1} 4)$ surface, which is in contrast to the omnipresence on metal substrates. ${ }^{[37-42]}$ Recalling the assumption of a templating effect due to a strong interaction between the protruding surface oxygen atoms and the molecular carboxylic acid groups, the reason for this absence is explained by the severe mismatch of the chicken-wire superstructure with the $\mathrm{CaCO}_{3}(10 \overline{1} 4)$ surface lattice as depicted in Figure 9. In this figure, the chicken-wire 

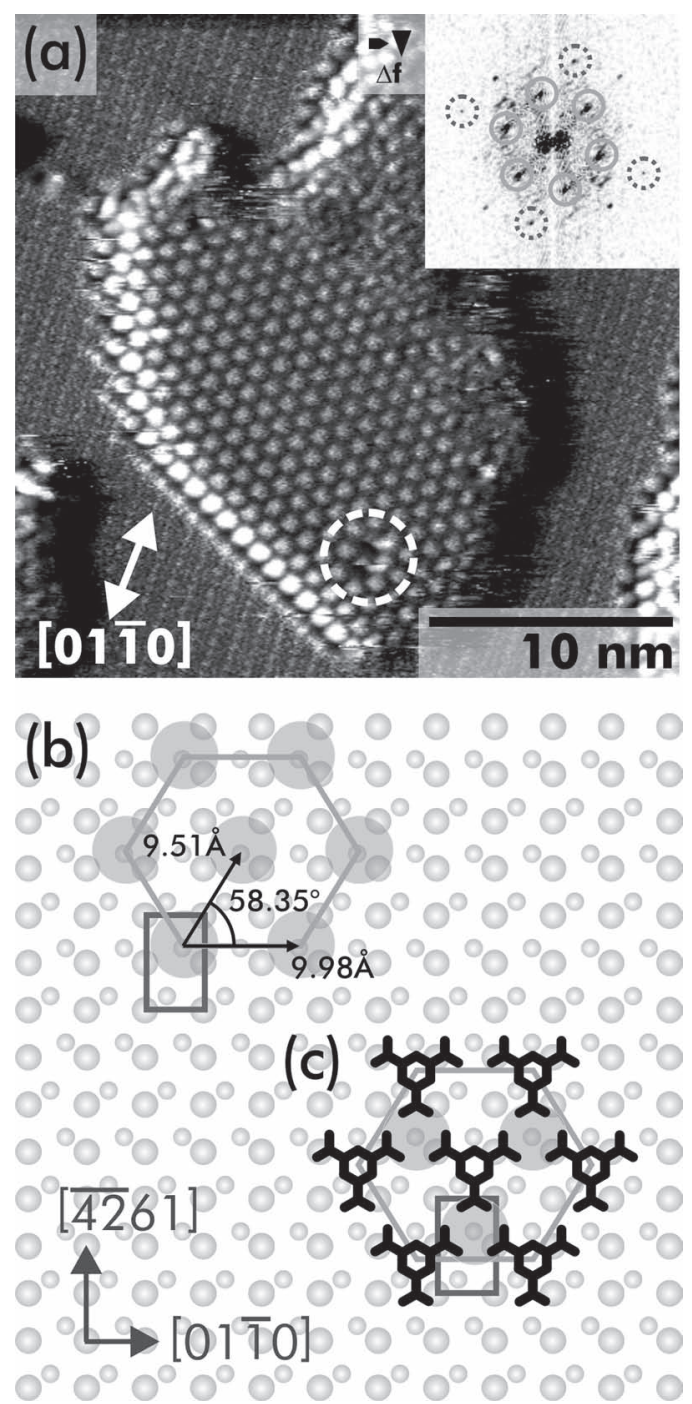

Figure 8. a) High-resolution $\Delta$ fimage of phase A. In the inset, the 2D-FT of (a) is shown. Spots originating from the molecular superstructure and from the calcite substrate are marked as solid circles and dotted circles, respectively. b,c) Model of the molecular adsorption structure stabilized by hydrogen bonds between the carboxylic groups of three flat-lying TMA molecules, resembling the flower motif. Due to templating by the underlying substrate, the resulting structure is not exactly hexagonal but slightly distorted, in excellent agreement with the experimental 2D-FT data.

motif is shown with dimensions derived from the bulk structure. A single TMA molecule can adsorb on the $\mathrm{CaCO}_{3}(10 \overline{1} 4)$ surface with all three carboxylic groups being in good registry with the surface lattice, most likely anchoring to protruding oxygen atoms. Upon extending the chicken-wire structure, however, the registry with the topmost oxygen atoms cannot be maintained, and thus, this adsorption geometry is expected to become energetically unfavorable.

Figure 10a and $\mathrm{b}$ represent high-resolution NC-AFM data acquired of phase $\mathrm{B}_{1}$. Additionally, high-resolution data of phase $\mathrm{C}$ embedded into a full monolayer of TMA molecules are presented (Figure 10c). For the phase $B_{1}$ and $B_{2}$, the molecular arrangement is most clearly revealed from the dissipation data reproduced in Figure 10b. The molecular

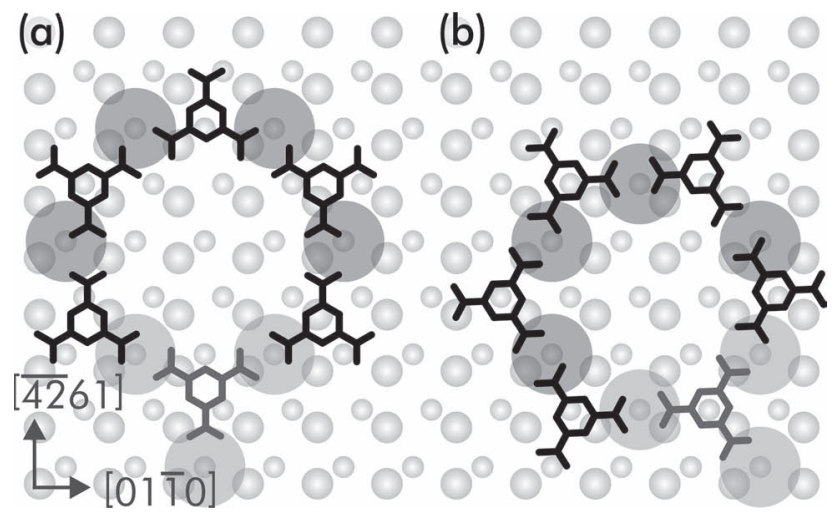

Figure 9. Chicken-wire motif of the TMA molecules superimposed to scale to the $\mathrm{CaCO}_{3}(10 \overline{1} 4)$ surface. While one molecule can bind to three surface oxygen atoms as suggested from the flower motif, an extended chicken-wire structure is incommensurable with the substrate structure. This is shown for two adsorption geometries in (a) and (b).

structure can be described by a center line (indicated as a dashed line in Figure 10a), to which additional molecules are attached on both sides. As already suggested by the data in Figure $7 \mathrm{~b}$, this center line draws an angle with respect to the [ $\overline{42} 61]$ direction. The two phases $B_{1}$ and $B_{2}$ differ by the sign of this angle. To be precise, an angle of $\pm(18.0 \pm 0.2)^{\circ}$ is revealed with respect to the [4261] direction. The existence of these two phases is in agreement with the glide plane symmetry of the $\mathrm{CaCO}_{3}(10 \overline{1} 4)$ surface.

The small $\mathrm{p} k_{\mathrm{a}}$ value of $3.12^{[34]}$ for TMA suggests that some of the TMA molecules might already deprotonate on the calcite surface at room temperature. This has been observed before for halide-substituted benzoic acid molecules having $\mathrm{p} k_{\mathrm{a}}$ values smaller than $3{ }^{[15]}$ As depicted in Figure 10d, the hydrogen-bond formation between carboxylic acid dimers requires protonated molecules; however, we cannot exclude the deprotonation of the outward oriented groups in Figure 10. Thus, in the case of TMA on calcite, the coexistence of the commensurate flower motif with fragments of the chickenwire illustrates the decisive influence of the substrate on the structure formation. In particular, substrate templating manifests itself here on the absence of extended chicken-wire networks.

\section{Conclusion}

In conclusion, the presented extended, hydrogen-bonded molecular structures prove to be stable on a bulk insulator at room temperature. TPA molecules on $\mathrm{CaCO}_{3}(10 \overline{1} 4)$ selfassemble into a $(2 \times 2)$ superstructure. This structure is characterized by molecular chains that align to form molecular ribbons. The structure of each chain agrees with the fundamental TPA bulk motif; however, the hydrogen bonds are stretched due to the presence of the surface. Moreover, the spacing of the molecular chains is dictated by the underlying substrate, enforcing a larger spacing compared to the molecular bulk structure.

Upon introducing a third carboxylic group as in TMA, the intermolecular and the molecular-surface interaction is 

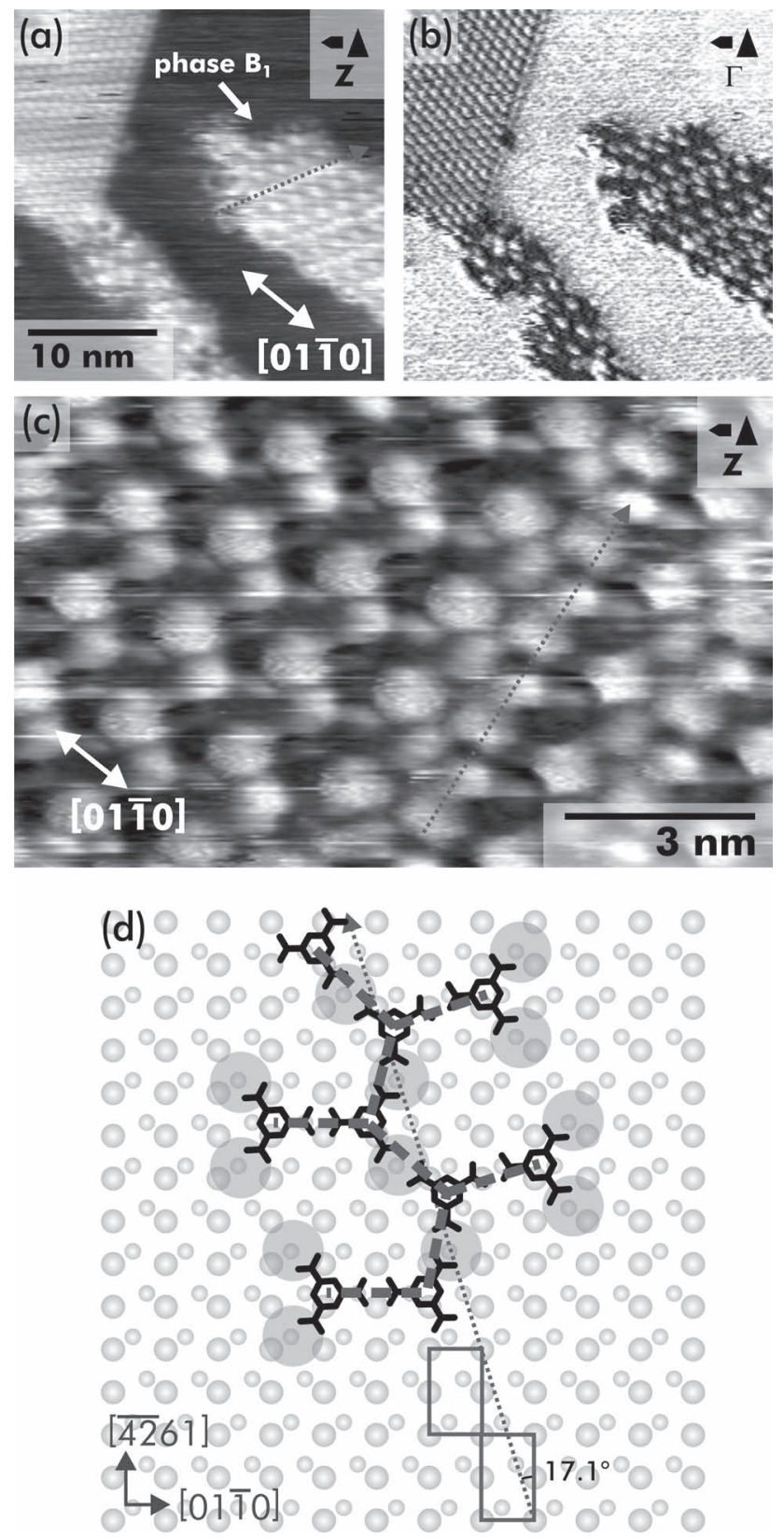

Figure 10. a,b) High-resolution NC-AFM images of the phase $B_{1}$. c) Phase $C$ revealed upon formation of a full monolayer. All observed motifs suggest a truncated chicken-wire motif. d) A structural model for this truncated structure.

enhanced. A well-ordered flower motif is revealed in this case that is composed by TMA molecules forming a nearly hexagonal pattern. A clear influence of the underlying substrate lattice is demonstrated by the distortion of the hexagonal pattern, which can be explained by a commensurate arrangement of the molecules with respect to the surface lattice. The absence of extended bulk-like structures readily manifests the influence of the substrate, as the bulk-like chicken-wire structure cannot be accommodated on $\mathrm{CaCO}_{3}(10 \overline{1} 4)$.

Our results demonstrate a significant substrate-templating effect being responsible for the structural variety observed upon molecular self-assembly on $\mathrm{CaCO}_{3}(10 \overline{1} 4)$. This study, thus, provides a strategy towards tailoring molecular structure formation on bulk insulator surfaces. Creating tunable molecular structures on insulating surfaces that remain stable at room temperature is of utmost importance for future molecular electronic devices and functional supramolecular assemblies.

\section{Experimental Section}

Sample preparation and NC-AFM experiments were carried out under ultra-high vacuum (UHV) conditions. Calcite crystals of optical quality were purchased from Korth Kristalle GmbH (Altenholz (Kiel), Germany) and cut mechanically to fit into the sample holder. After introduction into the UHV system, each crystal was freshly cleaved ${ }^{[44]}$ and annealed for about $1.5 \mathrm{~h}$ to a maximum temperature of about $450 \mathrm{~K}$. The latter strategy was followed to remove surface charges. A pristine crystal was used for each molecule deposition. The main surface orientation, which does not include the carbonate group orientation, was determined for each sample by measuring the unit cell dimensions in carefully driftcorrected $^{[43]}$ images.

The NC-AFM experiments were performed with an atomic force microscope (VT AFM 25 from Omicron, Taunusstein, Germany) operated in the frequency-modulated (FM) mode. ${ }^{[45]}$ In this mode, the change $\Delta f$ of the cantilevers resonance frequency upon tip-sample interaction is the main measurement signal. This signal is related to the tip-sample interaction force. ${ }^{[46,47]}$ The frequency shift $\Delta f$ was measured using a phase-locked loop controller (easyPLL plus from Nanosurf, Liestal, Switzerland).

Standard Si cantilevers (type PPP-NCH from Nanosensors, Neuchâtel, Switzerland) with resonance frequencies around $300 \mathrm{kHz}$ were excited to amplitudes around $10 \mathrm{~nm}$. All tips were initially bombarded with $\mathrm{Ar}^{+}$ions to remove contaminants and the oxide layer.

TPA and TMA molecules were purchased from Sigma Aldrich. TPA is specified with a quality of $\geq 99 \%$, while the best available quality for TMA was $\geq 97 \%$. The deposition is performed from a heated glass crucible. The initial deposition rate was determined with a quartz crystal microbalance. For TPA, at a temperature of $383 \mathrm{~K}$ a sublimation rate of about $0.03 \mathrm{ML} / \mathrm{min}$ was found, while TMA sublimates at $400 \mathrm{~K}$ at a rate of about $0.04 \mathrm{ML} / \mathrm{min}$. TPA has $\mathrm{p} k_{\mathrm{a}}$ values of 3.51 and 4.82 when dissociating the first and second carboxylic acid group, respectively. ${ }^{[34]}$ For TMA, p $k_{\mathrm{a}}$ values of 3.12, 3.89, and 4.7 have been determined for the first, second, and third dissociation, respectively. ${ }^{[34]}$ Compared with the deprotonation observed for some halogenated benzoic acids ${ }^{[15]}$ on calcite, no extensive deprotonation of the TPA and TMA molecules is expected herein because of the $\mathrm{p} k_{\mathrm{a}}$ values being larger than 3 .

NC-AFM data were analyzed and processed using the opensource software Gwyddion. ${ }^{[48]}$

\section{Supporting Information}

Supporting information is available from the Wiley Online Library or from the author. 


\section{Acknowledgements}

Financial support from the German Research Foundation (DFG) through grant KU1980/5-1 is gratefully acknowledged. We thank Jens Schütte and Ralf Bechstein for experimental support and most fruitful discussions.

[1] J. V. Barth, Annu. Rev. Phys. Chem. 2007, 58, 375.

[2] A. Kühnle, Curr. Opin. Colloid Interface Sci. 2009, 14, 157.

[3] W. Xu, M. Dong, H. Gersen, E. Rauls, S. Vazquez-Campos, M. Crego-Calama, D. N. Reinhoudt, I. Stensgaard, E. Lægsgaard, T. R. Linderoth, F. Besenbacher, Small 2007, 3, 854.

[4] W. Xu, M. D. Dong, H. Gersen, E. Rauls, S. Vazquez-Campos, M. Crego-Calama, D. N. Reinhoudt, E. Lægsgaard, I. Stensgaard, T. R. Linderoth, F. Besenbacher, Small 2008, 4, 1620.

[5] J. V. Barth, J. Weckesser, C. Cai, P. Günter, L. Bürgi, O. Jeandupeux, K. Kern, Angew. Chem. Int. Ed. 2000, 39, 1230.

[6] S. Maier, L.-A. Fendt, L. Zimmerli, T. Glatzel, O. Pfeiffer, F. Diederich, E. Meyer, Small 2008, 4, 1115.

[7] J. M. Mativetsky, S. A. Burke, S. Fostner, P. Grutter, Small 2007, 3, 818.

[8] T. Kunstmann, A. Schlarb, M. Fendrich, T. Wagner, R. Möller, R. Hoffmann, Phys. Rev. B 2005, 71, 121403.

[9] C. Bombis, N. Kalashnyk, W. Xu, E. Lægsgaard, F. Besenbacher, T. R. Linderoth, Small 2009, 5, 2177.

[10] S. A. Burke, W. Ji, J. M. Mativetsky, J. M. Topple, S. Fostner, H. J. Gao, H. Guo, P. Grütter, Phys. Rev. Lett. 2008, 100, 186104.

[11] T. Dienel, C. Loppacher, S. C. B. Mannsfeld, R. Forker, T. Fritz, Adv. Mater. 2008, 20, 959.

[12] R. Pawlak, L. Nony, F. Bocquet, V. Olson, M. Sassi, J. M. Debierre, C. Loppacher, L. Porte, J. Phys. Chem. C 2010, 114, 9290.

[13] P. Rahe, R. Lindner, M. Kittelmann, M. Nimmrich, A. Kühnle, Phys. Chem. Chem. Phys. 2012, 14, 6537.

[14] D. M. Duffy, J. H. Harding, J. Mater. Chem. 2002, 12, 3419.

[15] M. Kittelmann, P. Rahe, M. Nimmrich, C. M. Hauke, A. Gourdon, A. Kühnle, ACS Nano 2011, 5, 8420.

[16] P. Rahe, J. Schütte, A. Kühnle, J. Phys. Condens. Matter 2012, 24, 084006.

[17] J. Schütte, P. Rahe, L. Tröger, S. Rode, R. Bechstein, M. Reichling, A. Kühnle, Langmuir 2010, 26, 8295.

[18] S. L. Stipp, M. F. Hochella, Geochim. Cosmochim. Ac. 1991, 55 , 1723.

[19] S. L. S. Stipp, C. M. Eggleston, B. S. Nielsen, Geochim. Cosmochim. Ac. 1994, 58, 3023.

[20] O. M. Yaghi, M. O’Keeffe, N. W. Ockwig, H. K. Chae, M. Eddaoudi, J. Kim, Nature 2003, 423, 705.

[21] M. Lackinger, S. Griessl, L. Kampschulte, F. Jamitzky, W. M. Heckl, Small 2005, 1, 532.
[22] S. Stepanow, T. Strunskus, M. Lingenfelder, A. Dmitriev, H. Spillmann, N. Lin, J. V. Barth, C. Woell, K. Kern, J. Phys. Chem. B 2004, 108, 19392.

[23] S. Stepanow, M. Lingenfelder, A. Dmitriev, H. Spillmann, E. Delvigne, N. Lin, X. Deng, C. Cai, J. V. Barth, K. Kern, Nat. Mater. 2004, 3, 229.

[24] J. V. Barth, G. Costantini, K. Kern, Nature 2005, 437, 671.

[25] M. E. Cañas-Ventura, F. Klappenberger, S. Clair, S. Pons, K. Kern, H. Brune, T. Strunskus, C. Wöll, R. Fasel, J. V. Barth, J. Chem. Phys. 2006, 125, 184710.

[26] S. Clair, S. Pons, A. P. Seitsonen, H. Brune, K. Kern, J. V. Barth, J. Phys. Chem. B 2004, 108, 14585.

[27] P. Rahe, M. Nimmrich, A. Nefedov, M. Naboka, C. Wöll, A. Kühnle, J. Phys. Chem. C 2009, 113, 17471.

[28] J. S. Prauzner-Bechcicki, S. Godlewski, A. Tekiel, P. Cyganik, J. Budzioch, M. Szymonski, J. Phys. Chem. C 2009, 113, 9309.

[29] M. Bailey, C. J. Brown, Acta Cryst. 1967, 22, 387.

[30] A. Tekiel, J. S. Prauzner-Bechcicki, S. Godlewski, J. Budzioch, M. Szymonski, J. Phys. Chem. C 2008, 112, 12606.

[31] One monolayer is defined as two TPA molecules per $(2 \times 2)$ unit cell.

[32] Due to image distortions induced by thermal drift, the outlines are realigned with respect to the fixed features.

[33] S. Sadewasser, M. C. Lux-Steiner, Phys. Rev. Lett. 2003, 91, 266101.

[34] H. C. Brown, In Determination of Organic Structures by Physical Methods (Ed. E. A. Braude, F. C. Nachod), Academic Press: New YorkUSA, 1955.

[35] Note that the possibility of a double tip artifact in imaging singlemolecular chains can be ruled out due to the presence of singlemolecular defects (one is indicated in Figure 4a).

[36] D. J. Duchamp, R. E. Marsh, Acta Cryst. B 1969, 25, 5.

[37] J. M. MacLeod, O. Ivasenko, D. F. Perepichka, F. Rosei, Nanotechnology 2007, 18, 424031.

[38] M. Lackinger, S. Griessl, W. A. Heckl, M. Hietschold, G. W. Flynn, Langmuir 2005, 21, 4984.

[39] S. Griessl, M. Lackinger, M. Edelwirth, M. Hietschold, W. M. Heckl, Single Molecules 2002, 3, 25.

[40] A. Dmitriev, N. Lin, J. Weckesser, J. V. Barth, K. Kern, J. Phys. Chem. B 2002, 106, 6907.

[41] Y. C. Ye, W. Sun, Y. F. Wang, X. Shao, X. G. Xu, F. Cheng, J. L. Li, K. Wu, J. Phys. Chem. C 2007, 111, 10138.

[42] G. Sheerin, A. A. Cafolla, Surf. Science 2005, 577, 211.

[43] P. Rahe, R. Bechstein, A. Kühnle, J. Vac. Sci. Technol. B 2010, 28, C4E31.

[44] L. Tröger, J. Schütte, F. Ostendorf, A. Kühnle, M. Reichling, Rev. Sci. Instrum. 2009, 80, 063703.

[45] T. R. Albrecht, P. Grütter, D. Horne, D. Rugar, J. Appl. Phys. 1991, 69, 668.

[46] F. J. Giessibl, Phys. Rev. B 1997, 56, 16010.

[47] F. J. Giessibl, Rev. Mod. Phys 2003, 75, 949.

[48] D. Necas, P. Klapetek, Centr. Eur. J. Phys 2011, 10, 181.

Received: March 29, 2012

Revised: May 22, 2012

Published online: July 6, 2012 